\title{
An Atypical Presentation of Small Bowel Metastasis From a Previously Undiagnosed Primary Lung Adenocarcinoma
}

\author{
Andrew C. Berry ${ }^{\mathrm{a}, \mathrm{h}}$, Rachel E. Herdes ${ }^{\mathrm{a}}$, Marcelo F. Larsen ${ }^{\mathrm{b}}$, Andres J. Yarur ${ }^{\mathrm{b}}$, James S. Scolapio ${ }^{\mathrm{c}}$, \\ Brijesh B. Patel ${ }^{\mathrm{d}}$, Danny Avalos ${ }^{\mathrm{e}}$, Joshua D. Lenchus ${ }^{\mathrm{f}}$, Warren L. Reuther III ${ }^{\mathrm{g}}$
}

\begin{abstract}
Lung adenocarcinoma commonly metastasizes to the liver, brain, bones and adrenal glands, with metastasis to the small bowel in only $0.5-2 \%$ of cases. Not only did our patient have metastases to the small bowel, but the patient presented solely with gastrointestinal symptoms and no evidence of lung disease clinically or on imaging. Immunohistochemical analysis helped diagnose a primary lung adenocarcinoma with metastasis to the small bowel. There are few reported cases of small bowel metastases leading to the diagnosis of a primary lung cancer, let alone, in living patients. Though uncommon, physicians should not dismiss the possibility of vague gastrointestinal symptoms as a manifestation of metastases from a primary lung cancer.
\end{abstract}

Keywords: Small intestine; Pathology; Histopathology; Lung cancer

\section{Introduction}

Lung cancer is the leading cause of neoplastic death, with

Manuscript accepted for publication July 24, 2014

${ }^{a}$ Kansas City University of Medicine and Biosciences (KCUMB), Kansas City, MO, USA

${ }^{b}$ Department of Medicine, Division of Gastroenterology, University of Miami Miller School of Medicine, Miami, FL, USA

${ }^{c}$ Department of Medicine, Division of Gastroenterology, University of Florida, Jacksonville, FL, USA

${ }^{\mathrm{d}}$ Department of Internal Medicine, University of South Florida, Tampa, FL, USA

${ }^{e}$ Department of Internal Medicine, University of Miami Miller School of Medicine Palm Beach Regional Campus, Atlantis, FL, USA

${ }^{\mathrm{f}}$ Department of Internal Medicine, University of Miami Miller School of Medicine, Miami, FL, USA

${ }^{g}$ Department of Radiology, West Palm Hospital, West Palm Beach, FL, USA

${ }^{\mathrm{h}}$ Corresponding Author: Andrew C. Berry, Kansas City University of Medicine and Biosciences, 1750 E. Independence Ave, Kansas City, MO 64106, USA. Email: ABerry5555@gmail.com

doi: http://dx.doi.org/10.14740/jmc1874w reported survival rates of $20 \%$ at 1 year and $1 \%$ at 5 years after diagnosis $[1,2]$. It is responsible for more deaths than the next three most common cancers combined (colon, breast and prostate) [3]. Non-small cell lung cancer (NSCLC) accounts for the majority of lung neoplasias and includes squamous cell carcinomas, adenocarcinomas and large cell carcinomas. Adenocarcinoma is the most common type of lung cancer in people under the age of 45 years. It commonly metastasizes to the liver, brain, bones and adrenal glands, with $85 \%$ of patients having metastasis at the time of diagnosis [1]. Primary lung cancer rarely metastasizes to the small bowel and is only seen in about $0.5-2 \%$ of cases $[4,5]$. We describe a patient who presented solely with gastrointestinal symptoms, which was later found to be the result of metastasis from an undiagnosed primary NSCLC (adenocarcinoma).

\section{Case Report}

A 49-year-old Caucasian female presented to the emergency department with intractable bilious vomiting and abdominal cramping. She had been treated for a partial small bowel obstruction 4 weeks prior to admission with temporary resolution of symptoms. During the month prior to admission, the patient developed daily emesis and experienced a 17-pound unintentional weight loss. Her past medical and surgical histories are significant only for the partial small bowel obstruction, a volvulus at age 25, peptic ulcer disease, chronic alcoholism, anemia, asthma, one C-section and a left Achilles tendon rupture. She had a 75-pack-year smoking history. Past alcohol history was significant for 12 alcoholic drinks per day for the last 3 - 4 years, although she has been alcohol free for the last 5 weeks. Upon admission, she was afebrile, tachycardic at 112 , respiratory rate of 18 , blood pressure of $110 / 76$ and oxygen saturation of $96 \%$ on room air. Physical exam was pertinent for hyperactive bowel sounds and significant tenderness to palpation in all four abdominal quadrants. Laboratory values were within normal limits except: platelet count $(132,000$ per $\mu \mathrm{L})$, sodium $(121 \mathrm{mmol} / \mathrm{L})$, potassium $(2.8 \mathrm{mmol} / \mathrm{L})$, chloride $(77 \mathrm{mmol} / \mathrm{L})$, total alkaline phosphatase $(140 \mathrm{IU} / \mathrm{L})$.

Chest X-ray was unremarkable and showed no acute 


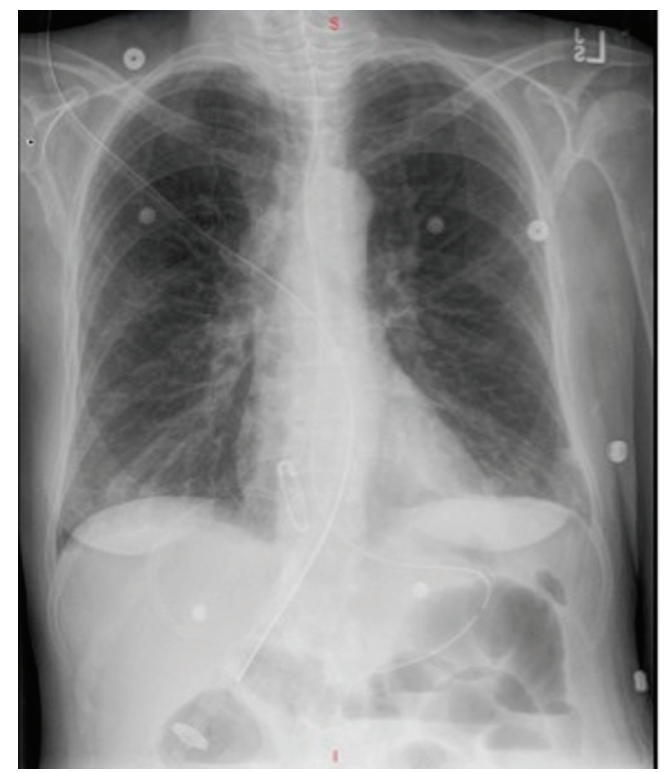

Figure 1. Posterior/anterior (PA) chest X-ray on day of admission showing no acute disease and normal pulmonary vascularity.

disease (Fig. 1). CT of abdomen and pelvis without contrast revealed a high-grade small bowel obstruction, with maximum small bowel diameter of $5.5 \mathrm{~cm}$ significantly increased from her previous imaging 4 weeks ago (Fig. 2). Abdominal ultrasound revealed fatty infiltration of the liver and multiple fluid-filled dilated small bowel loops. Small bowel resection (12 inches), gastrojejunostomy and appendectomy were performed. The patient underwent small bowel resection and pathology revealed poorly differentiated metastatic adenocarcinoma most consistent with a pulmonary primary malignancy (Fig. 3). Long-term intubation, tracheostomy and PEG tube placement were done. The patient later displayed signs of tamponade amid a large pericardial effusion, and

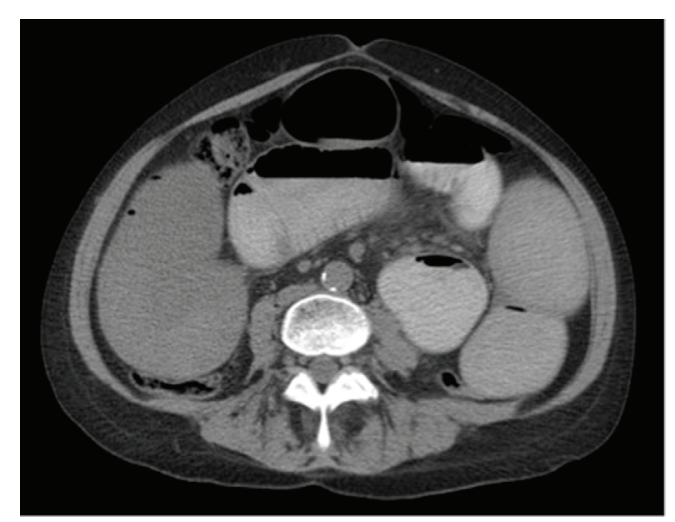

Figure 2. CT of abdomen and pelvis without contrast showing multiple dilated loops of small bowel throughout the abdomen with compression of the loops comparable with a high-grade small bowel obstruction.
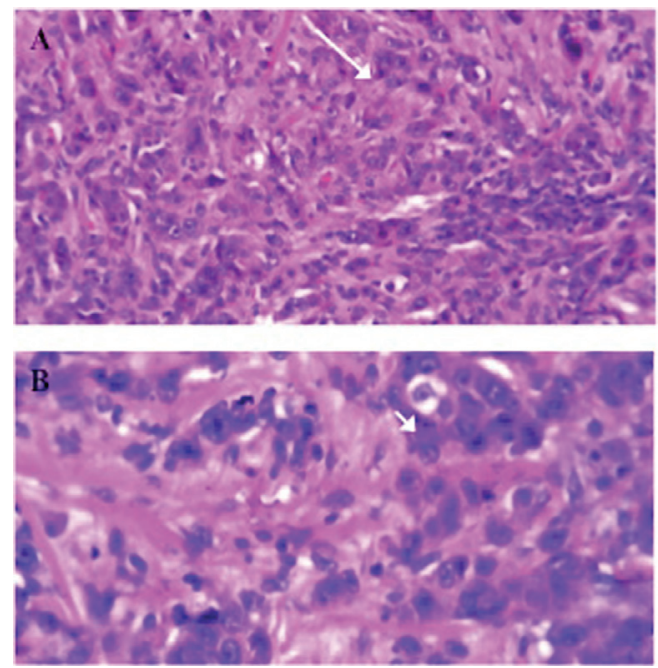

Figure 3. (A) H\&E stain of invasive carcinoma into the small bowel submucosa with atypical mitosis (arrow). (B) H\&E stain of the small bowel demonstrating undifferentiated adenocarcinoma with glandular formation (arrow head).

pericardiocentesis was performed with immunohistochemistry (IHC) staining revealing metastatic adenocarcinoma consistent with lung primary (Fig. 4). Less than 1 month after diagnosis and 2 months after first presentation of symptoms, the patient passed away.

\section{Discussion}

Gastrointestinal metastasis from lung cancer is rare with a reported incidence of $0.5-2.0 \%[4,5]$. It is even more uncommon in living patients, as its presence usually indicates advanced disease and poor prognosis. Lung cancer commonly metastasizes to regional lymph nodes (72-84\%), liver (33$47 \%$ ), bones (21-34\%), brain (16-32\%), adrenals (20-29\%) and heart (12-29\%) [6, 7]. When gastrointestinal metastases do occur, it usually presents asymptomatically because it remains only in the submucosal layer [8]. Patients typically present with vomiting, abdominal pain, anemia, and/or melena as the lesions grow and cause ulcerations and obstruction [8]. Small bowel metastases can also be revealed by peritonitis or small bowel obstructions [9]. Gastrointestinal metastases from a primary lung cancer most commonly occur in the esophagus. Though uncommon, small bowel metastases from a primary lung cancer occur in the ileum more frequently than the jejunum [10]. Small bowel metastases can occur in every type of primary lung cancer; however, undifferentiated large cell and small cell carcinoma are most prevalent [11].

Metastasis to the small bowel following diagnosis of a primary lung cancer is rare, yet there have been even fewer reported cases of small bowel metastases leading to the di- 


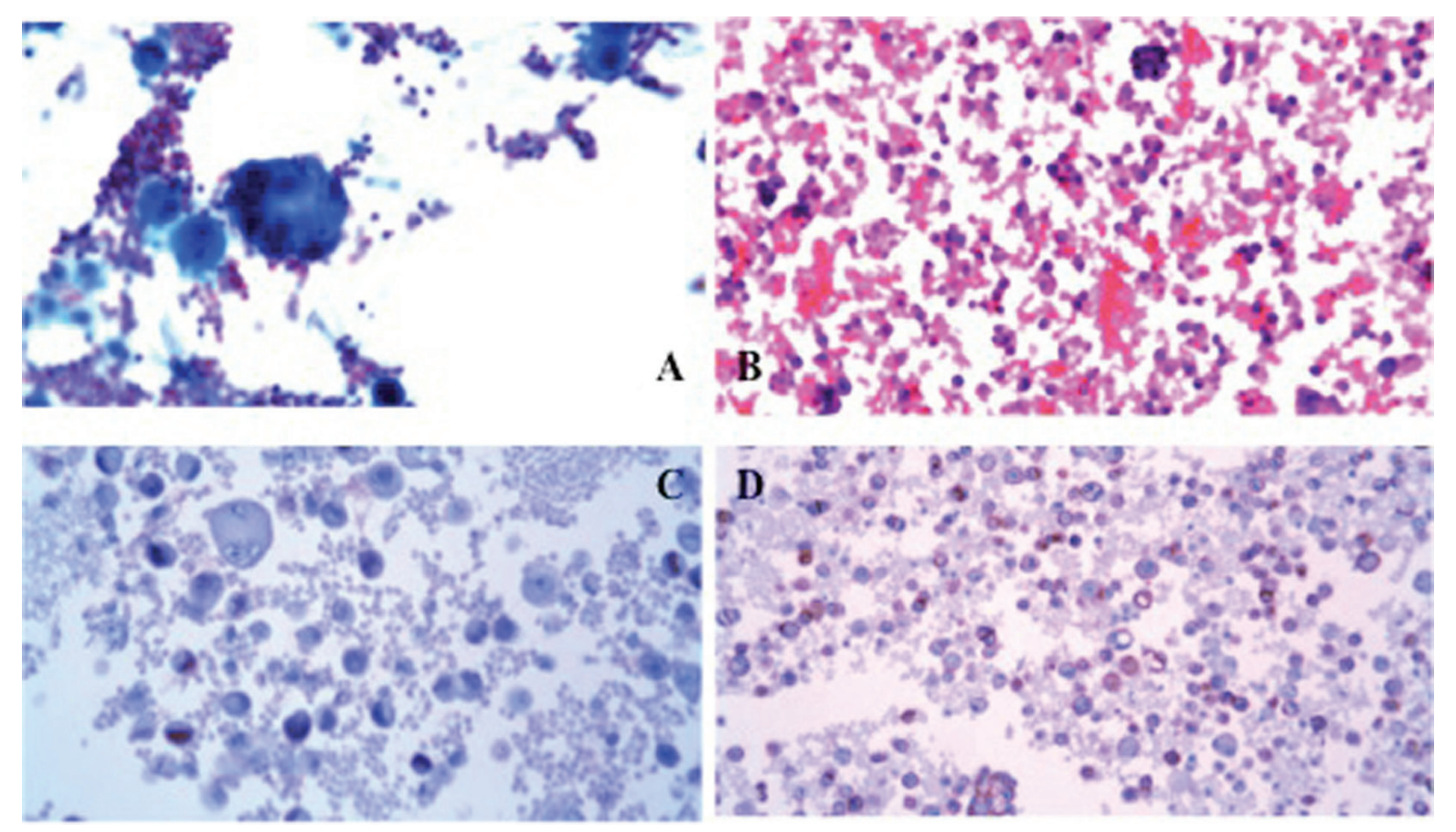

Figure 4. (A) Pericardial fluid aspiration showing glandular structures. (B) Tumor cells within the pericardial fluid aspirate. (C) Immunohistochemical stain positive for TTF-1 (lung and thyroid cancer marker). (D) Immunohistochemical stain positive for MOC-31 (adenocarcinoma marker).

agnosis of a primary lung cancer, let alone, in living patients $[9,12]$. A couple cases have been reported of small bowel perforation leading to the diagnosis of small bowel metastasis from a previously undiagnosed lung cancer [12]. This case is unique because there was initially no evidence of any lung disease clinically and on imaging (chest X-ray) and the patient presented solely with vague gastrointestinal symptoms: vomiting, abdominal cramping and nonspecific weight loss without any respiratory symptoms. IHC analysis of the small bowel resection helped confirm metastatic malignancy. Positive TTF-1 and CK7 stains plus negative CK20 stain, suggested metastatic adenocarcinoma, poorly differentiated, from a primary lung adenocarcinoma [13]. In addition, pericardial fluid IHC not only confirmed the diagnosis but also helped differentiate general metastases from a primary lung cancer. Further staging for extrapulmonary metastases is unknown as a PET scan was not performed. Most cases of gastrointestinal metastases have multiple metastatic sites, with an average of 4.8 sites [11]. However, a recent case report by Rivera et al has described the first reported case of solitary metastasis to the small bowel [4]. Although solitary metastasis is a possibility in our case, it would be a very atypical presentation, and hard to confirm without a PET scan.

Gastrointestinal metastasis from a primary lung cancer has a very poor survival rate $[9,14,15]$. The median time from lung cancer diagnosis to gastrointestinal metastasis was 3 months and the average time from diagnosis of GI metastasis to death was 2.8 months $[14,15]$. Studies have also reported that survival time is independent of initial diagnosis tumor stage $[14,15]$. Therefore, surgical resection should be considered as only a palliative option to help prevent further peritonitis, bowel obstruction or small bowel perforation [12]. Clinicians should always keep in mind gastrointestinal symptoms as a manifestation of lung cancer metastasis, and as in our case, small bowel metastasis leading to diagnosis of a primary lung cancer.

\section{Acknowledgement}

We would like to acknowledge Dr. Wayne Cai, M.D., of West Palm Hospital in West Palm Beach, FL, USA, for his pathological assistance.

\section{Grant Support}

No financial support was obtained for the project. No financial grant was obtained. None of the authors listed received any financial support or services or any other contributions for their work.

\section{References}

1. Yang CJ, Hwang JJ, Kang WY, Chong IW, Wang TH, 
Sheu CC, Tsai JR, et al. Gastro-intestinal metastasis of primary lung carcinoma: clinical presentations and outcome. Lung Cancer. 2006;54(3):319-323.

2. Greenlee RT, Hill-Harmon MB, Murray T, Thun M. Cancer Statistics, 2001. CA: A Cancer Journal for Clinicians. 2001;51:15-36.

3. American Cancer Society. Cancer facts and figures. Atlanta:American Cancer Society; 2013.

4. Rivera NT, Katz H, Weisbaum G, Guarneri R, Bray N, Constanza-Guaqueta D. Solitary Metastasis to the Small Bowel from Primary Adenocarcinoma of the Lung. J Gastrointest Cancer. 2013.

5. Antler AS, Ough Y, Pitchumoni CS, Davidian M, Thelmo W. Gastrointestinal metastases from malignant tumors of the lung. Cancer. 1982;49(1):170-172.

6. Steinhart AH, Cohen LB, Hegele R, Saibil FG. Upper gastrointestinal bleeding due to superior mesenteric artery to duodenum fistula: rare complication of metastatic lung carcinoma. Am J Gastroenterol. 1991;86(6):771774 .

7. Stenbygaard LE, Sorensen JB, Larsen H, Dombernowsky P. Metastatic pattern in non-resectable nonsmall cell lung cancer. Acta Oncol. 1999;38(8):993-998.

8. Okazaki R, Ohtani H, Takeda K, Sumikawa T, Yamasaki A, Matsumoto S, Shimizu E. Gastric metastasis by primary lung adenocarcinoma. World J Gastrointest Oncol.
2010;2(10):395-398.

9. Berger A, Cellier C, Daniel C, Kron C, Riquet M, Barbier JP, Cugnenc PH, et al. Small bowel metastases from primary carcinoma of the lung: clinical findings and outcome. Am J Gastroenterol. 1999;94(7):1884-1887.

10. De Castro CA, Dockerty MB, Mayo CW. Metastatic tumors pf the small intestine. Surg Gynec Obs. 1957;105:159-165.

11. McNeill PM, Wagman LD, Neifeld JP. Small bowel metastases from primary carcinoma of the lung. Cancer. 1987;59(8):1486-1489.

12. Leidich RB, Rudolf LE. Small bowel perforation secondary to metastatic lung carcinoma. Ann Surg. 1981;193(1):67-69.

13. Su YC, Hsu YC, Chai CY. Role of TTF-1, CK20, and CK7 immunohistochemistry for diagnosis of primary and secondary lung adenocarcinoma. Kaohsiung J Med Sci. 2006;22(1):14-19.

14. Goh BK, Yeo AW, Koong HN, Ooi LL, Wong WK. Laparotomy for acute complications of gastrointestinal metastases from lung cancer: is it a worthwhile or futile effort? Surg Today. 2007;37(5):370-374.

15. Lee PC, Lo C, Lin MT, Liang JT, Lin BR. Role of surgical intervention in managing gastrointestinal metastases from lung cancer. World J Gastroenterol. 2011;17(38):4314-4320. 